\title{
PAPERS
}

\section{The human two domain trefoil protein, TFF2, is glycosylated in vivo in the stomach}

\author{
F E B May, J I Semple, J L Newton, B R Westley
}

\begin{abstract}
Background-TFF2, a member of the trefoil factor family (TFF) of peptides, is a secreted protein of $\mathbf{1 0 6}$ amino acids that is expressed in mucous neck cells of the fundus and glands at the base of the antrum in normal human stomach. TFF 2 is also detected at high concentrations around sites of ulceration. It is protective against mucosal damaging agents and stimulates cell motility.
\end{abstract}

Aims-To measure the expression of TFF2 in normal human stomach and its secretion into gastric juice.

Methods-TFF2 cDNA was amplified by reverse transcription polymerase chain reaction from gastric mucosa and sequenced. Gastric juice or cytosol, prepared from gastric mucosa, was obtained from individuals with macroscopically normal stomachs. TFF2 concentrations were measured by quantitative western transfer analysis.

Results-Sequencing of TFF2 cDNA revealed a single amino acid change from the published sequence. Significant amounts of $12 \mathrm{kDa}$ TFF2 were detected in human gastric juice. Larger quantities of a protein of higher apparent molecular mass were also detected. This was shown to be N-glycosylated TFF2 using the endoglycosidase, peptide-N-Gycosidase $\mathrm{F}$. The majority of TFF2 in normal gastric mucosa was also glycosylated.

Conclusions-Human TFF2 is glycosylated via an $\mathrm{N}$-linkage, presumably on Asn $^{15}$ which forms part of the single consensus site for $\mathbf{N}$-glycosylation in human TFF2. The glycosylation may be of functional significance. Future studies of human TFF2 should use antibodies raised against the correct amino acid sequence. Biological studies should be performed with recombinant protein of the correct sequence, and the biological consequences of glycosylation investigated.

(Gut 2000;46:454-459)

Keywords: TFF peptide; stomach; glycosylation; TFF2; restitution

TFF2 was the second member of the human trefoil factor family (TFF) of peptides to be identified. ${ }^{1}$ Three human trefoil peptides have been identified, whose corresponding genes are clustered on chromosome 21, with TFF1 12.5 $\mathrm{kb}$ upstream of $T F F 2$, which is approximately $40 \mathrm{~kb}$ upstream of TFF3. ${ }^{2}{ }^{3}$ Trefoil peptides are small, secreted proteins, characterised by a conserved domain of 42-43 amino acid residues. ${ }^{34}$ The six conserved cysteine residues of the trefoil domain (see fig 1A) form three intramolecular disulphide bonds and hence three loops that are stacked in a characteristic three loop structure..$^{5-8}$ Mature TFF2 is a 12 $\mathrm{kDa}$ protein of 106 amino acids and contains two trefoil domains separated by a short sequence of seven residues. ${ }^{1}$ The structure of the porcine homologue of TFF2 has been solved by both $x$ ray crystallography and solution nuclear magnetic resonance. ${ }^{5-7}$ It is a relatively elongated molecule, consisting of two compact globular domains that adopt the same tertiary structure, joined via a small interface. A striking feature of both trefoil domains is a surface patch formed from five conserved residues that have no obvious structural role. The two patches are located at the far ends of the protein molecule. ${ }^{5-7}$

TFF2 is expressed predominantly by Brunner's glands in the duodenum and in the normal human gastric antrum. ${ }^{19}$ High concentrations of TFF2 mRNA are found in cells adjacent to sites of mucosal ulceration in the gastrointestinal tract. ${ }^{10}$ This prompted speculation that trefoil peptides have a role in tissue repair. ${ }^{3411}$ Following acute mucosal injury, repair of the mucosa of the gastrointestinal tract is initiated within a few minutes. One of the earliest processes is a rapid migration of cells from the margins of the damaged region over the denuded area to re-establish epithelial continuity, a process called epithelial restitution. TFF 2 may be of particular interest among trefoil peptides as it is produced within 30 minutes after cryoprobe induction of gastric ulceration in rats. ${ }^{12}$

Studies with recombinant TFF2 produced in Sacchromyces cerevisiae ${ }^{13}$ have shown that $\mathrm{TFF} 2$ protects rats from both indomethacin and restraint induced gastric mucosal damage, and ethanol induced damage. ${ }^{14}{ }^{15}$ In vitro, TFF2 stimulated human colonic carcinoma

Abbreviations used in this paper: $\mathrm{TFF}$, trefoil factor family. 

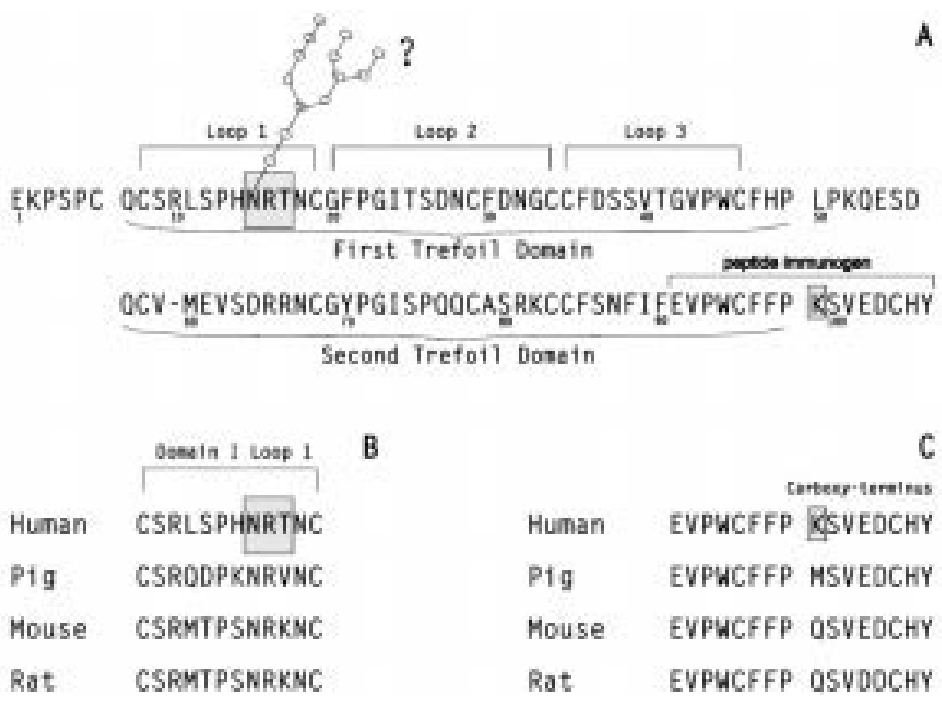

Figure 1 Sequence of TFF 2 protein. (A) Sequence of the mature TFF2 protein derived from TFF 2 mRNA from human gastric mucosa of three individuals and the TFF2 gene. The positions of the two trefoil domains are indicated as are the amino acids included in each of the three loops formed during folding of the trefoil domains. The single

$N$-glycosylation recognition sequence (NRT) which is a potential glycosylation site located in loop 1 of the first trefoil domain is boxed. The lysine residue $(K)$ which replaces the asparagine residue found in the previously published sequence at position 99 is also boxed. The 16 amino acid peptide synthesised for use as an immunogen is overlined. Spaces have been inserted in the sequence after residues 6, 49, and 98 to indicate the limits of the trefoil domains. (B) The amino acid sequences of the first loop in domain I of the four known mammalian TFF2 proteins: human TFF2 $2^{1}$ (present study); porcine TFF $2^{25}$; murine

$T_{F F} 2^{26}$; and rat TFF $2{ }^{27}$ (C) Comparison of the 16 amino acid residues at the carboxy termini of the four known mammalian TFF2 protein sequences proteins: human TFF2 (present study); porcine TFF ${ }^{25}$; murine TFF $2^{26}$; and rat TFF $2 .{ }^{27}$

cells to migrate across and invade into collagen gel, ${ }^{14}$ and stimulated their migration across plastic in an in vitro wounding assay. ${ }^{14} 16$

In the present study, we have examined the expression of human TFF2 in gastric juice and in normal gastric mucosa.

\section{Methods}

COLLECTION AND PREPARATION OF HUMAN SAMPLES

Samples were collected from subjects undergoing diagnostic endoscopy for investigation of anaemia, dysphagia, or abdominal pain. None had significant past medical history or were taking acid suppressive or non-steroidal antiinflammatory medication. Gastric juice was collected at the time of gastroscopy from nine subjects with macroscopically normal stomachs. Samples were aspirated via a suction unit using a standard in continuity cytology trap. Cytosol was prepared from human gastric mucosa. Four gastric antral biopsy specimens were collected at the time of gastroscopy from each of five subjects with macroscopically normal stomachs. All specimens were taken using standard endoscopic biopsy forceps from within $2 \mathrm{~cm}$ of the pylorus, and stored frozen at $-70^{\circ} \mathrm{C}$. Four antral specimens from each subject were homogenised in a glass:glass homogeniser in inhibitor buffer: $1 \mathrm{mM}$ iodoacetamide, $4 \mathrm{mM}$ phenylmethlysulphonyl fluoride, $5 \mathrm{mM}$ benzamidine $\mathrm{HCl}, 10 \mathrm{mM}$ EDTA, $100 \mathrm{mM} \alpha$ aminocaproic acid, $10 \mathrm{mM}$ N-ethyl maleimide, $67 \mathrm{mM}$ sodium phosphate $\mathrm{pH}$ 6.5. Sample 1 was homogenised in $1 \mathrm{ml}$ whereas samples 2-5 were homogenised in $300 \mu$ of inhibitor buffer. After centrifugation for one hour at $100000 \mathrm{~g}$, the cytosol was stored at $-70^{\circ} \mathrm{C}$. The protein concentrations of the juice and cytosols were measured using the bicinchoninic acid protein assay. ${ }^{17}$ Cytosol was also prepared from normal gastric mucosa, distant from any lesions, taken from three gastrectomy specimens.

For RNA preparation, samples of normal gastric mucosa were taken from gastrectomy specimens of patients with gastric carcinoma. Total RNA was extracted from the precipitate obtained after homogenisation of the tissue in $\mathrm{LiCl}$ and urea as described previously. ${ }^{18}$

Ethical permission for the collection of human material used in this study was obtained from the South of Tyne Health Authority Ethical Committee. All subjects gave written informed consent.

ISOLATION AND SEQUENCING OF TFF2 CDNA AND GENOMIC DNA

TFF2 cDNA was obtained by reverse transcription polymerase chain reaction (RT-PCR) from total stomach RNA and cloned in the plasmid pEZZ18 (Pharmacia). The primers (5'-GAG AAACCCTCCCCCTGCCAGTGC-3' and 5'TTAGTAATGGCAGTCTTCCAC-3') amplified the sequence between nucleotides 71 and 392 of the TFF 2 mRNA (numbering according to Tomasetto and colleagues ${ }^{1}$ ). Five recombinants from each of three stomachs were sequenced using a sense primer (5'GAAAGTAGACGCGA-3') and the -40 mer primer, the sequences of which are located in the vector and Sequenase 2.0 (USB/Amersham).

\section{ISOLATION AND SEQUENCING OF TFF2 GENOMIC} DNA

A library of human placental DNA, partially digested with $M b o I$, was constructed using standard techniques in the $\lambda$ Dash replacement vector. The library was plated on Escherichia coli P2PLK and screened unamplified using a ${ }^{32} \mathrm{P}$ labelled TFF2 cDNA probe as described previously. ${ }^{2}$ The TFF2 probe was obtained by RT-PCR of RNA extracted from human gastric mucosa. The primers (5'-CCCAG ATCCTGGCAGCGC-3' and 5'-TTTCTT GGTTTCGGAACACCCG-3') amplified the sequence between nucleotides 23 and 447 of the TFF2 mRNA (numbering according to Tomasetto and colleagues ${ }^{1}$ ). The PCR product was cloned into the Sma I site of Bluescript KSM13 (Stratagene) and a cDNA probe prepared from the recombinant plasmid by PCR. The library was screened, the recombinants that had hybridised were plaque purified, and the $\lambda \mathrm{DNA}$ prepared from them as described previously. ${ }^{2}$

DNA from two $\lambda$ recombinants was sequenced using Sequenase 2.0 (USB/ Amersham) and the primer (5'-CTC TCGGAAGTGCTGCTTCTCC-3') from nucleotides 309 to 330 (numbering according to Tomasetto and colleagues ${ }^{1}$ ).

PRODUCTION OF TFF2 SPECIFIC ANTISERA

A peptide corresponding to the 16 carboxy terminal amino acid residues of human TFF2 (see fig 1A) was synthesised by Fmoc polyamide chemistry, purified by high pressure liquid 
chromatography, and its authenticity verified by matrix assisted laser desorption mass spectrometry. The peptide was conjugated to bovine serum albumin prior to immunisation of rabbits and to keyhole limpet haemocyanin prior to immunisation of $\mathrm{BALB} / \mathrm{c}$ mice. Rabbits were immunised with $200 \mu \mathrm{g}$ and mice were immunised with $50 \mu \mathrm{g}$ of conjugated peptide. Initial injections were in alum and subsequent injections were in phosphate buffered saline. ${ }^{19}$

POLYACRYLAMIDE GEL ELECTROPHORESIS

Gels were prepared using the acrylamide ratios and buffers described previously. ${ }^{20}$ The separating gels used for the transfers shown in figs 2 and 4 contained a gradient of $10-32 \%(\mathrm{wt} / \mathrm{vol})$ polyacrylamide, and for the transfer shown in fig 3 contained a gradient of $10-35 \%$ (wt/vol) polyacrylamide; the stacking gels contained 5\% (wt/vol) polyacrylamide. Sodium dodecyl sulphate (SDS) was included in the loading buffer at a final concentration of $2 \%(\mathrm{wt} / \mathrm{vol}) .{ }^{21} \mathrm{Sam}-$ ples to be fractionated were brought to 62.5 $\mathrm{mM}$ Tris $\mathrm{HCl} \mathrm{pH} 6.8,12.5 \mathrm{mM}$ EDTA, 100 $\mathrm{mM} \quad \beta$ mercaptoethanol, and $0.005 \%$ bromophenol blue and boiled for five minutes prior to loading. Molecular weight markers and known amounts of recombinant TFF2 were included on all the gels.

Recombinant TFF2 protein with a lysine at position 99 was produced in Pichia pastoris using an expression vector that directs secretion of the recombinant protein into the culture media. The medium was harvested and TFF2 purified by cation exchange chromatography, anion exchange chromatography, and lectin affinity chromatography. Both glycosylated and non-glycosylated mature TFF 2 were produced and purified. The mass of the nonglycosylated, purified recombinant TFF2 was determined by matrix assisted laser desorption mass spectrometry and was within $0.02 \%$ of the calculated molecular mass. For quantification of TFF2, known amounts of recombinant TFF2 and glycosylated TFF2 were electrophoresed alongside the human samples. The glycosylated recombinant TFF2 migrates in the same position as naturally occurring human glycosylated TFF2. The intensities of the reactive bands were measured using image analysis. Only non-glycosylated recombinant TFF2 was electrophoresed in the gels shown in figs 2 and 4.

\section{WESTERN TRANSFER}

Samples were transferred from the gel to a polyvinylidene difluoride (PVDF) membrane, $0.2 \mu \mathrm{m}$ pore size (Schleicher and Schuell) using a semidry transfer apparatus (Schleicher and Schuell) for 15 minutes at $100 \mathrm{~mA}$ and then left uncovered at room temperature overnight. $^{21}$ They were fixed in $0.2 \%$ glutaraldehyde for 45 minutes, blocked with $3 \%$ (wt/vol) bovine serum albumin in phosphate buffered saline, and then incubated with $1 / 2000$ dilution of polyclonal anti-TFF 2 in 3\% (wt/vol) bovine serum albumin and phosphate buffered serum for two hours at $37^{\circ} \mathrm{C}$. They were then incubated for a further two hours at $37^{\circ} \mathrm{C}$ with a secondary antibody conjugated to alkaline phosphatase in phosphate buffered saline containing $0.1 \%$ Tween 20 and developed as described previously. ${ }^{19}$

DEGLYCOSYLATION OF HUMAN TFF2 FROM GASTRIC JUICE

Aliquots $(10 \mu \mathrm{l})$ of gastric juice 1 were incubated for one hour at $37^{\circ} \mathrm{C}$ in the presence or absence of peptide-N-Gycosidase F (Boehringer Mannheim) as recommended by the manufacturer. Incubations were in the absence and presence of the protease inhibitor pepstatin.

\section{Results}

SEQUENCE OF TFF2 AND PRODUCTION OF TFF2 SPECIFIC ANTIBODIES

Prior to raising human TFF2 specific antibodies, we isolated and sequenced TFF2 mRNA from the gastric mucosa of three individuals to confirm the protein sequence published by Tomasetto et al. ${ }^{1}$ RNA was prepared from gastric mucosa taken from three individuals. TFF2 cDNA corresponding to the mature TFF2 protein was amplified from the RNA using TFF2 specific primers. The PCR products were either sequenced directly or cloned in plasmid vectors prior to sequencing. Two discrepancies from the sequence of Tomasetto and colleagues ${ }^{1}$ were found consistently. The codon for the conserved $\mathrm{Arg}^{65}$ (see fig 1) in domain II is CGA, not AGA. This is a conservative change. The codon for residue 99 which is in the carboxy terminal part of the protein outside the second trefoil domain is AAG, not AAC. This means that the amino acid residue in that position is a lysine and not an asparagine as reported originally. ${ }^{1}$ The presence of a lysine at position 99 was confirmed by sequencing exon 3 of the TFF2 gene isolated from a human placental DNA library. Figure $1 \mathrm{~A}$ gives the complete sequence of mature human TFF2.

A peptide corresponding to the 16 carboxy terminal amino acids, with a lysine residue at the equivalent of position 99, was synthesised. Following conjugation to bovine serum albumin and keyhole limpet haemocyanin, polyclonal antisera were raised in rabbits and mice, respectively. The reactivities of the antisera were evaluated by their ability to react with the TFF2 peptide used as the immunogen.

DETECTION OF TFF2 IN GASTRIC JUICE

To determine whether we could use the TFF2 antisera to measure expression of TFF2, samples of gastric juice were analysed following western transfer. Known amounts of recombinant TFF2 of the correct molecular mass were electrophoresed through the polyacrylamide gel. As shown in fig 2, a protein band of apparent molecular mass of approximately 12 $\mathrm{kDa}$ that comigrated with recombinant TFF2 was detected clearly in six of the nine samples of gastric juice. It seems therefore that this less abundant TFF2 immunoreactive protein band corresponds to mature, secreted TFF2.

In all nine samples of gastric juice, the major TFF2 immunoreactive protein band was a more slowly migrating protein of apparent 


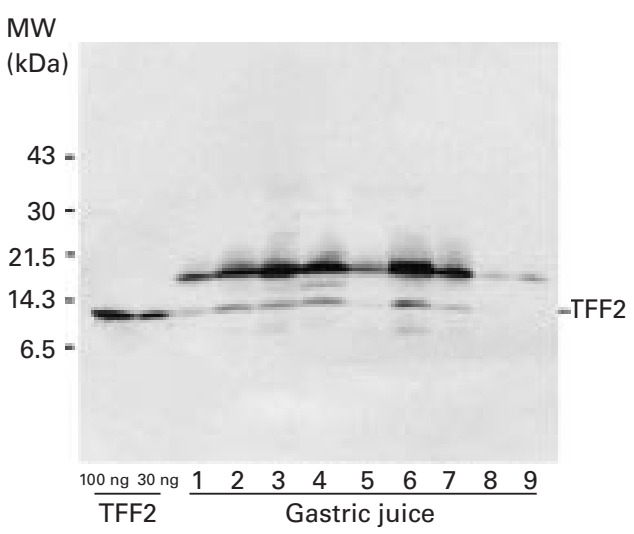

Figure 2 Detection of TFF2 in human gastric juice. Aliquots $(10 \mu \mathrm{l})$ of gastric juice were subjected to electrophoresis on a denaturing polyacrylamide gel, transferred to a PVDF membrane, and reacted with TFF2 antisera. Known amounts of recombinant TFF2 were also analysed. The positions of the molecular mass markers $(M W)$ are shown on the left and of TFF2 on the right hand side.

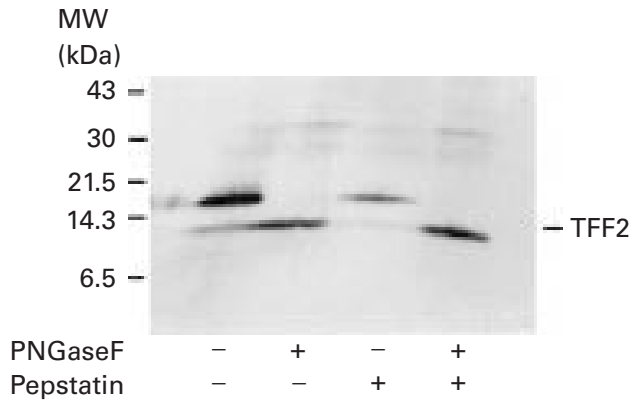

Figure 3 Human TFF2 is glycosylated via an N-linkage. Aliquots of gastric juice were denatured and incubated under denaturing conditions in the absence or presence of peptide-N-Gycosidase F (PNGase F), and in the absence or presence of pepstatin. TFF2 was detected by western transfer analysis. The position of TFF2 is shown on the right hand side of the gel and of the molecular weight markers on the left hand side.

molecular mass of approximately $20 \mathrm{kDa}$. The nature of the larger immunoreactive protein band was considered. Prior to electrophoresis, the proteins were boiled with the thiol agent $\beta$ mercaptoethanol and the ionic detergent SDS; it is unlikely therefore that the larger protein band represents a complex between TFF2 and another molecule. It could be a related protein that cross reacts with the TFF2 antisera, an incorrectly processed precursor of mature $\mathrm{TFF} 2$, or $\mathrm{TFF} 2$ protein that has undergone some post-translational modification.

Table 1 Concentration of TFF2 in human gastric juice

\begin{tabular}{lclll}
\hline Sample & $\begin{array}{c}\text { Glycosylated } \\
\text { TFF2 }(\mu \mathrm{g} / \mathrm{ml})\end{array}$ & TFF2 $(\mu \mathrm{g} / \mathrm{ml})$ & $\begin{array}{l}\text { Total TFF2 } \\
(\mu \mathrm{g} / \mathrm{mg} \text { protein })\end{array}$ & $\begin{array}{l}\text { Ratio of glycosylated TFF2: } \\
\text { non-glycosylated TFF2 }\end{array}$ \\
\hline GJ 1 & 3.0 & 0.6 & 3.6 & $5: 1$ \\
GJ 2 & 9.0 & 1.5 & 10.5 & $6: 1$ \\
GJ 3 & 15.0 & 1.5 & 16.5 & $9: 1$ \\
GJ 4 & 14.0 & 2.0 & 16.0 & $7: 1$ \\
GJ 5 & 2.4 & 0.3 & 2.7 & $8: 1$ \\
GJ 6 & 20.0 & 3.0 & 23.0 & $6.3: 1$ \\
GJ 7 & 10.0 & 1.0 & 11.0 & $10: 1$ \\
GJ 8 & 1.0 & 0.1 & 1.1 & $10: 1$ \\
GJ 9 & 1.5 & ND & 1.5 &
\end{tabular}

GJ, gastric juice; ND, not detected.

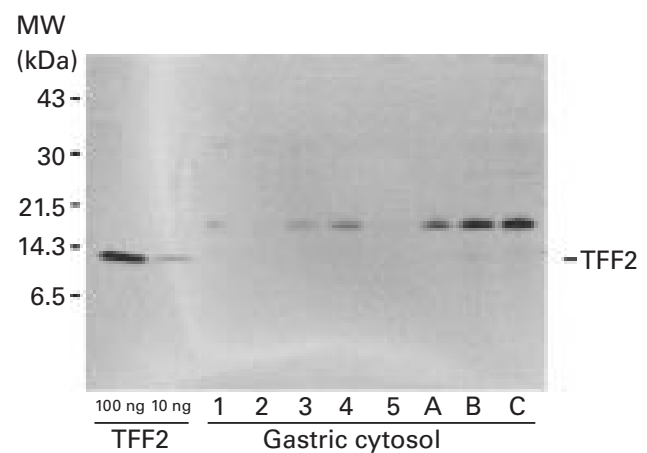

Figure 4 Detection of TFF2 in cytosol prepared from human gastric mucosa by western transfer analysis. Cytosol was prepared from small biopsy specimens of normal human gastric mucosa (samples 1-5), or mucosa from gastrectomy specimens (samples $A-C$ ). The positions of the molecular mass markers (MW) are shown on the left and of TFF2 on the right hand side.

HUMAN TFF2 IS GLYSOSYLATED IN VIVO

There is a single recognition sequence for $\mathrm{N}$-linked glycosylation in TFF2 within loop 1 of the first trefoil domain, $\mathrm{N}^{15} \mathrm{R}^{16} \mathrm{~T}^{17}$ (see fig 1A). The possibility that TFF2 is glycosylated via an $\mathrm{N}$-linkage in vivo, and that the larger protein band represents glycosylated TFF2, was tested with the endoglycosidase, peptide$\mathrm{N}$-Gycosidase F, which removes N-linked glycans from glycoproteins by cleaving between the asparagine residue and the first sugar which is always an $\mathrm{N}$-acetyl-D-glucosamine. The proteins in gastric juice sample 1 were first denatured and then incubated in the absence and presence of peptide-N-Gycosidase $\mathrm{F}$ before separation by gel electrophoresis, transfer to PVDF, and immunodetection with TFF2 antiserum. Duplicate incubations were performed in the presence of pepstatin, to ensure that proteolysis during the incubation did not confound interpretation. The result shown in fig 3 confirms that the major TFF2 immunoreactive protein band in gastric juice sample 1 is larger than mature $\mathrm{TFF} 2$. Incubation with peptide-N-Gycosidase F converted the slower migrating TFF2 immunoreactive band to a form that comigrated with mature TFF2. This shows that the larger TFF2 immunoreactive protein band contains an $\mathrm{N}$-linked oligosaccharide and that human TFF2 is secreted as a glycosylated protein in vivo.

Table 1 shows the concentrations of glycosylated and non-glycosylated TFF2 in the nine samples of gastric juice. The total TFF2 concentrations varied over a 20-fold range from 1 to over $20 \mu \mathrm{g} / \mathrm{ml}$, and its concentration as a percentage of total protein varied 10 -fold from $0.07 \%$ to $0.7 \%$. In all nine samples of gastric juice, glycosylated $\mathrm{TFF} 2$ was the major form of TFF2 detected. The ratio of glycosylated to non-glycosylated TFF2 varied between 5:1 and 10:1 (see table 1).

TFF2 IN HUMAN GASTRIC MUCOSA

To investigate which form of TFF2 is present in the gastric mucosa, cytosol was prepared from eight samples of antral mucosa: five collected during gastroscopy and three from gastrectomy specimens. The proteins in cytosol extracts were separated by denaturing gel elec- 
trophoresis and transferred to PVDF; the TFF2 protein was detected as described in the Methods. Figure 4 shows that glycosylated $\mathrm{TFF} 2$ is the major form of TFF2 in gastric mucosa. This indicates that the majority of TFF2 synthesised and secreted from the gastric mucosa is glycosylated. The ratio of glycosylated TFF2 to non-glycosylated TFF2 seems to be higher in the mucosa than in the gastric lumen which may suggest that following secretion, some becomes deglycosylated in the gastric lumen. TFF2 was barely detectable in normal human gastric mucosa samples 2 and 5 and was only visible on the original transfer membrane.

\section{Discussion}

The identification of a lysine residue at position 99 of TFF2 in material prepared from four individuals, instead of the asparagine residue reported, suggests that either this represents a frequent polymorphism or that there was an error in the original sequence. ${ }^{1}$ Replacement of an amino acid that has a relatively short, polar side chain by an amino acid with a long, positively charged side chain would increase the $\mathrm{pI}$ of the protein and may have effects on its structure and susceptibility to proteolysis. In agreement with our findings, another group identified a potential polymorphism of TFF $2 .^{22}$

The carboxy terminal sequences of the four known mammalian TFF2 proteins are compared in fig $1 \mathrm{C}$. There is high sequence conservation in this region, with only one other amino acid residue showing variation; residue 102 is glutamic acid in human, porcine, and murine TFF2, whereas it is aspartic acid in rat TFF2. Residue 99 is the most variant: either lysine or asparagine in human TFF2, methionine in porcine TFF2, and glutamine in rodent TFF2. Clearly these are not conservative changes, and if residue 99 is involved in the biological function of TFF2 then these substitutions are likely to have an effect.

It is difficult to predict what effect the substitution of a lysine residue for an asparagine residue will have on the structure of human TFF2, especially as it is outside the more highly structured trefoil domains. Studies on the functional activity of TFF2 published to date have used a recombinant human TFF2 with an asparagine residue at position 99. It will be interesting to investigate whether TFF2 with a lysine residue at position 99 has the same activities or whether this substitution is of biological significance. It is possible that the relatively high concentrations of TFF2 required to obtain an effect in some studies are owing to the substitution of an asparagine residue.

Many studies on the expression of TFF2 have used antibodies raised against a peptide corresponding to the 16 carboxy terminal residues of human TFF2. Residue 99 lies in the middle of this peptide, and this may affect the specificity of the antibodies raised. We have synthesised a 16 residue peptide with a lysine residue at the equivalent of position 99. The antisera produced react specifically with TFF2 and do not cross react with TFF1, or any TFF3 that might be present, in the stomach or gastric juice (see figs 2, 3, and 4).

HIGH CONCENTRATIONS OF TFF2 ARE PRESENT IN HUMAN GASTRIC JUICE

The concentration of TFF2 in gastric juice varies between 1 and $23 \mu \mathrm{g} / \mathrm{ml}$, with a mean value of $9.5(2.6) \mu \mathrm{g} / \mathrm{ml}$. All patients had fasted prior to endoscopy and it is probable that these values reflect a natural variation between individuals. This is considerably higher than the 0.03 to $0.1 \mu \mathrm{g} / \mathrm{ml}$ reported for the concentration of TFF1 in gastric juice from six individuals. ${ }^{23}$ It is interesting that the concentration of TFF2 in gastric juice is so much higher than the concentration of TFF1. Such a difference may be suggestive of significant functional differences between the two proteins.

HUMAN TFF2 IS A GLYCOSYLATED PROTEIN IN VIVO

The finding that TFF2 is predominantly a glycosylated protein in vivo was unexpected. In gastric tissue only the glycosylated form was detected, and in gastric juice, glycosylated TFF2 was present in an excess of between 5:1 and 10:1 to non-glycosylated TFF2. We show here that the glycosylation is via an N-linkage. When recombinant human TFF2 was expressed in $S$ cerevisiae, a significant proportion of the recombinant protein was glycosylated via an N-linkage on $\mathrm{Asn}^{15} \cdot{ }^{13}$ However at the time, natural TFF2 had not been studied at the protein level, and as $S$ cerevisiae is known to produce recombinant mammalian proteins that are glycosylated aberrantly, it was not assumed that natural TFF2 was glycosylated. The next year, in a paper reporting the production of an anti-TFF2 monoclonal antibody, an immunoreactive protein band of the predicted size of non-glycosylated mature TFF2 was detected in extracts from normal stomach, duodenum, and ileum but not colon. ${ }^{24}$ The reasons for this discrepancy with the present study are unclear, but it did establish the view that TFF2 is not normally glycosylated. ${ }^{3424}$ Our finding that TFF2 is glycosylated in 17 individuals now reverses this misconception.

There is no evidence that TFF 1 or TFF 3 are glycosylated, nor do they contain consensus sequences for $\mathrm{N}$-glycosylation. The glycosylation of TFF2 is a potentially important difference between the three human trefoil proteins.

The effect of this glycosylation on the structure and biological activity of TFF2 must now be considered. Asn ${ }^{15}$ is in the first loop of the first trefoil domain of TFF2, immediately adjacent to the most highly conserved amino acid residue $\mathrm{Arg}^{16}$. Indeed $\mathrm{Arg}^{16}$ is the central residue in the three amino acid consensus sequence for N-linked glycosylation of $\mathrm{Asn}^{15}$. $\mathrm{Arg}^{16}$ is a structurally important residue thought to be critical in stabilising the core structure between the first and third loops. In porcine TFF2, residues $12-16$ were found to form a $3_{10}$ helix $\mathrm{C}$ in crystals ${ }^{5}$ and to be part of a turn in solution. ${ }^{7}$ In a second paper, on the $x$ ray crystallographic structure of porcine $\mathrm{TFF} 2$, 
TFF2 was present as an asymmetric unit, as a dimer. The authors detected the formation of two hydrogen bonds between $A s n^{15}$ and $\mathrm{Asn}^{67}$, and of van der Waals contacts involving Lys ${ }^{14}$ $\mathrm{Asn}^{15}$ and $\mathrm{Asn}^{67}-\mathrm{Pro}^{71}{ }^{6}$ It seems probable that the presence of an $\mathrm{N}$-linked oligosaccharide on Asn ${ }^{15}$ will affect the stability of these structural elements and inter-residue contacts.

The amino acid sequence of the first loop of domain I of human TFF2 that contains the consensus recognition sequence for $\mathrm{N}$-glysosylation is compared with the corresponding sequences of the other three known TFF2 proteins in fig $1 \mathrm{~B} .{ }^{13}{ }^{25-27}$ In the tripeptide consensus sequence, both the asparagine that is glycosylated and the arginine are conserved but the threonine is variant, being replaced by a valine in porcine TFF2 and lysine in the rodent TFF 2 proteins. Although both murine and rat TFF 2 contain a threonine at position 12 , these threonine residues do not form part of an $\mathrm{N}$-glycosylation consensus recognition sequence and there are no other N-glycosylation consensus sequences in the proteins. ${ }^{2627}$ All mammalian TFF2 proteins are expressed in the stomach, principally in the antrum, and in the duodenum. Porcine, rat, and murine TFF2 are also expressed in the pancreatic acinar cells, ${ }^{42324}$ whereas human TFF2 is not. ${ }^{4}$ Pancreatic TFF2 presumably provides an additional source of TFF2 that is biologically active in the gastrointestinal tract distal to the stomach. It would be interesting if human TFF2 was not expressed by the pancreas because the TFF2 produced by the human stomach was more active or stable than that of other mammals as a result of its glycosylation.

Clearly the effect of glycosylation on the biological activity of TFF2 also needs to be considered. It is possible that glycosylation could affect the half life of TFF2. Playford et al have observed that glycosylated recombinant TFF2 is more potent than non-glycosylated TFF2 in vivo in a rat model of protection against gastric damage. ${ }^{14}$ The biological activities of glycosylated and non-glycosylated TFF2 now need to be compared rigorously in a range of biological assays.

This work was supported by the Cancer Research Campaign. JIS thanks the Cancer Research Campaign for a research studentship and JLN thanks the Astra Foundation for a clinical research fellowship. We are grateful to Professor OFW James and A Allen for advice and helpful discussions.

1 Tomasetto C, Rio M-C, Gautier C, et al. hSP, the human homolog of pS2 protein is co-expressed with pS2 in stomach but not in breast carcinoma. EMBO f 1990;9:407-14.

2 May FEB, Westley BR. Close physical linkage of the genes encoding the pNR-2/pS2 protein and human spasmolytic protein (hSP). Hum Genet 1997;99:303-7.
3 Thim L. Trefoil peptides: from structure to function. Cell Mol Life Sci 1997;53:888-903.

4 Poulsom R. Trefoil peptides. Baillieres Clin Gastroenterol 1996;10:113-34.

5 Gajhede M, Petersen TN, Henriksen A, et al. Pancreatic spasmolytic polypeptide (PSP): first three-dimensional structure of a member of the mammalian trefoil family of peptides. Structure 1993;1:253-62.

6 De A, Brown DG, Gorman MA, et al. Crystal structure of a disulphide-linked "trefoil" motif found in a large family of putative growth factors. Proc Natl Acad Sci USA 1994;91: 1084-8.

7 Carr MD, Bauer CJ, Gradwell MJ, et al. Solution structure of a trefoil-motif-containing cell growth factor, porcine of a trefoil-motif-containing cell growth factor, porcine 2206-10.

8 Polshakov VI, Williams MA, Gargaro AR, et al. Highresolution solution structure of human pNR-2/pS2: a single trefoil motif protein. $\mathcal{F}$ Mol Biol 1997;267:418-32.

9 Hanby AM, Poulsom R, Singh S, et al. Spasmolytic polypeptide is a major antral peptide: distribution of the trefoil peptides human spasmolytic polypeptide and pS2 in the stomach. Gastroenterology 1993;105:1110-16.

10 Rio M-C, Chenard, M-P, Wolf C, et al. Induction of pS2 and hSP genes as markers of mucosal ulceration of the digestive tract. Gastroenterology 1991;100:375-9.

11 May FEB, Westley BR. Trefoil proteins: their role in normal and malignant cells. $\mathcal{F}$ Pathol 1997;183:4-7.

12 Alison MR, Chinery R, Poulsom R, et al. Experimental ulceration leads to sequential expression of spasmolytic polypeptide, intestinal trefoil factor, epidermal growth factor, and transforming growth factor alpha mRNAs in rat stomach. F Pathol 1995;175:405-14.

13 Thim L, Norris K, Norris F, et al. Purification and characterisation of the trefoil peptide human spasmolytic polypeptide (hSP) produced in yeast. FEBS Lett 1993;318: 345-52.

14 Playford RJ, Marchbank T, Chinery R, et al. Human spasmolytic polypeptide is a cytoprotective agent that stimulates cell migration. Gastroenterology 1995;108:108-16.

15 Babyatsky MW, deBeaumont M, Thim L, et al. Oral trefoil peptides protect against ethanol- and indomethacinnduced gastric injury in rats. Gastroenterology 1996;110: 489-97.

16 Dignass A, Lynch-Devaney K, Kindon H, et al. Trefoil peptides promote epithelial migration through a transforming growth factor $\beta$-independent pathway. $\mathcal{F}$ Clin Invest 1994;94:376-83.

17 Smith PK, Krohn RI, Hermanson GT, et al. Measurement of protein using bicinchoninic acid. Anal Biochem 1985; 150:76-85.

18 May FEB, Westley BR. Cloning of estrogen-regulated messenger RNA sequences from human breast cancer cells. Cancer Res 1986;46:6034-40.

19 Piggott NH, Henry JA, May FEB, et al. Antipeptide antibodies against the pNR-2 oestrogen-regulated protein of human breast cancer cells and detection of pNR-2 expression in normal tissues by immunohistochemistry. $\mathcal{F}$ Pathol 1991;63:95-104.

20 Westley BR, Knowland JS. An estrogen receptor from Xenopus laevis liver possibly connected with vitellogenin synthesis. Cell 1978;15:367-74

21 Chadwick MP, Westley BR, May FEB. Homodimerization and hetero-oligomerization of the single-domain trefoil protein $\mathrm{pNR}-2 / \mathrm{pS} 2$ through cysteine 58. Biochem ff 1997;327:117-23.

22 Sieb T, Blin N, Hilgert K, et al. The three human trefoil genes $\mathrm{TFF} 1, \mathrm{TFF} 2$ and TFF3 are located within a region of $55 \mathrm{~kb}$ on chromosome 21q22.3. Genomics 1997;40:200-2.

23 Rio MC, Bellocq JP, Daniel JY, et al. Breast cancerassociated pS2 protein: synthesis and secretion by normal associated pS2 protein: synthesis and secr

24 Elia G, Chinery R, Hanby AM, et al. The production and characterisation of a new monoclonal antibody to the trefoil peptide human spasmolytic polypeptide. Histochem $\mathcal{F}$ 1994;26:644-7

25 Thim L, Thomsen J, Christen M, et al. The amino acid sequence of pancreatic spasmolytic polypeptide. Biochim Biophys Acta 1985;827:410-18.

26 LeFebvre $\mathrm{O}$, Wolf $\mathrm{C}$, Kédinger $\mathrm{M}$, et al. The mouse one P-domain (pS2) and two P-domain (mSP) genes exhibit distinct patterns of expression. F Cell Biol 1993;122:191-8.

27 Jeffrey GP, Oates PS, Wang TC, et al. Spasmolytic polypeptide: a trefoil peptide secreted by rat gastric mucous cells. Gasteoenterology 1994;106:336-45. 